\title{
A Case of Cefepime-Induced Neurotoxicity: Renal Function Missing in Action
}

\author{
Brooke P. Lichak ${ }^{1}$, Omolara Lawal ${ }^{2}$, Hyma V. Polimera ${ }^{1}$, Ashwani Garg ${ }^{1}$, Gurwant Kaur ${ }^{3}$ \\ 1. Internal Medicine, Penn State Health Milton S. Hershey Medical Center, Hershey, USA 2. Neurology, Penn State \\ Health Milton S. Hershey Medical Center, Hershey, USA 3. Nephrology, Penn State Health Milton S. Hershey Medical \\ Center, Hershey, USA
}

Corresponding author: Brooke P. Lichak, blichak@pennstatehealth.psu.edu

\begin{abstract}
Cefepime is a renally excreted, fourth-generation cephalosporin used in the treatment of severe abdominal, skin, soft tissue, and urinary tract infections due to its broad-spectrum coverage. Cefepime-induced neurotoxicity is a rare but serious side effect that has increased in recent years likely due to increased antibiotic use, increased drug resistance, and increased symptom recognition. While decreased glomerular filtration rate is an important risk factor for developing elevated serum cefepime levels, recent literature has suggested that a significant proportion of patients with normal renal function can also develop neurotoxicity from cefepime. Here, we present a case of cefepime-induced neurotoxicity to demonstrate the importance of monitoring mental status changes in all patients being treated with cefepime.
\end{abstract}

Categories: Internal Medicine, Infectious Disease, Nephrology

Keywords: cefepime-induced neurotoxicity, cefepime, neurotoxicity, encephalopathy, acute kidney injury, toxicity, seizure, acute interstitial nephritis, acute tubular necrosis

\section{Introduction}

Cefepime-induced neurotoxicity has a wide range of symptoms including impaired consciousness, encephalopathy, myoclonus, seizures, and coma [1]. Risk factors include decreased glomerular filtration rate (GFR), high drug dosing, and dysfunction of the blood-brain barrier. The primary proposed mechanism of cefepime-induced neurotoxicity is competitive inhibition of $\gamma$-amino butyric acid receptors in the central nervous system [2]. This suppression of the primary inhibitory receptor in the central nervous system likely lowers the threshold of excitation which subsequently can result in seizures or global encephalopathy. Due to the increasing incidence of cefepime-induced neurotoxicity and prevalence in healthy individuals, it is imperative for physicians to have a high index of suspicion for neurotoxic effects when treating patients with cefepime. By closely monitoring a patient's renal function, adverse consequences can be recognized quickly and managed appropriately. This article was previously presented as a poster at the Society of Hospital Medicine South Central Pennsylvania Chapter annual meeting on October 17, 2020.

Review began 01/24/2021 Review ended 02/13/2021 Published 02/16/2021

\section{(c) Copyright 2021}

Lichak et al. This is an open access article distributed under the terms of the Creative Commons Attribution License CC-BY 4.0., which permits unrestricted use, distribution, and reproduction in any medium, provided the original author and source are credited.

\section{Case Presentation}

A 59-year-old female with a history of neuromyelitis optica with baseline paraplegia and neurogenic bladder on chronic foley presented to the emergency department with altered mental status.

She was recently admitted to the hospital with septic shock requiring pressors for one day secondary to resistant Escherichia coli urinary tract infection and acute kidney injury (AKI) with elevated creatinine of 1.4 $\mathrm{mg} / \mathrm{dL}$ (baseline of 0.6-0.7 mg/dL approximately five months ago) and decreased creatinine clearance $(\mathrm{CrCl})$ of $36 \mathrm{~mL} / \mathrm{min}$. Her renal function quickly improved on day two of admission to a creatinine of $0.7 \mathrm{mg} / \mathrm{dL}$ and $\mathrm{CrCl}$ of $56.5 \mathrm{~mL} / \mathrm{min}$. She was initially started on intravenous (IV) ceftriaxone and later changed to IV cefepime due to her urine culture, sensitivity pattern, and renal recovery (Figure 1). A PICC line was placed and the patient was discharged with IV cefepime. She had completed a 10-day course of cefepime just one day prior to the current admission. 


\section{Cureus}

\begin{tabular}{|l|c|c|c|c|}
\hline & \multicolumn{2}{|c|}{ Escherichia coli } & \multicolumn{2}{c|}{ Escherichia coli \#2 } \\
\hline Drug & Broth & Broth MIC & Broth & Broth MIC \\
\hline Amoxicillin/Clavulanic A & Resistant & $>16 / 8$ & Suscept. & $8 / 4$ \\
\hline Ampicillin & Resistant & $>16$ & Resistant & $>16$ \\
\hline Ampicilin/Sulbactam & Intermed. & $16 / 8$ & Intermed. & $16 / 8$ \\
\hline Aztreonam & Suscept. & $<=4$ & Suscept. & $<=4$ \\
\hline Cefazolin & Resistant & $>16$ & Suscept. & $<=2$ \\
\hline Cefepime & Suscept. & $<=2$ & & \\
\hline Cefotaxime & Resistant & 8 & & \\
\hline Cefotetan & Intermed. & 32 & & \\
\hline Ceftazidime & Resistant & 16 & & \\
\hline Ceftriaxone & Resistant & 8 & & \\
\hline Ciprofloxacin & Resistant & $>2$ & Resistant & $>2$ \\
\hline Ertapenem & Suscept. & $<=0.5$ & Suscept. & $<=0.5$ \\
Gentamicin & Suscept. & $<=1$ & Suscept. & $<=1$ \\
\hline Levofloxacin & Resistant & $>4$ & Resistant & $>4$ \\
\hline Meropenem & Suscept. & $<=1$ & & \\
\hline Nitrofurantoin & Intermed. & 64 & Suscept. & $<=32$ \\
\hline Piperacillin Tazobac & Suscept. & $<=8$ & Suscept. & $<=8$ \\
\hline Tetracycline & Suscept. & $<=4$ & Suscept. & $<=4$ \\
Trimeth-Sulfamethoxazole & Suscept. & $<=2 / 38$ & Suscept. & $<=2 / 38$ \\
\hline
\end{tabular}

FIGURE 1: Urine culture and sensitivity report on first admission.

During this admission, initial vitals were temperature $35.8^{\circ} \mathrm{C}$, heart rate 68 beats per minute, respiratory rate 19 breaths per minute, and blood pressure $81 / 54 \mathrm{mmHg}$. Her systolic blood pressures were in 80 s for the last two to three months prior to admission and was maintained on home midodrine. This was attributed to autonomic dysfunction secondary to neuromyelitis optica after extensive workup. Examination was notable for expressive aphasia and altered mental status. She was also found to have AKI with elevated creatinine of $2.19 \mathrm{mg} / \mathrm{dL}$ (baseline $0.6 \mathrm{mg} / \mathrm{dL}$ ), blood urea nitrogen of $68 \mathrm{mg} / \mathrm{dL}$ (baseline $7 \mathrm{mg} / \mathrm{dL}$ ), and estimated glomerular filtration rate (eGFR) of $23 \mathrm{~mL} / \mathrm{min} / 1.73 \mathrm{~m}^{2}$ (baseline $>60 \mathrm{~mL} / \mathrm{min} / 1.73 \mathrm{~m}^{2}$ ). Electrolyte levels were serum sodium $145 \mathrm{mmol} / \mathrm{L}$ (baseline $141 \mathrm{mmol} / \mathrm{L}$ ), serum potassium $4.4 \mathrm{mmol} / \mathrm{L}$ (baseline $4.3 \mathrm{mmol} / \mathrm{L}$ ), serum chloride $108 \mathrm{mmol} / \mathrm{L}$ (baseline $104 \mathrm{mmol} / \mathrm{L}$ ), and serum bicarbonate $18 \mathrm{mmol} / \mathrm{L}$ (baseline $26 \mathrm{mmol} / \mathrm{L}$ ). Her urine output was decreased to $600 \mathrm{~mL}$ in 24 hours. Brain computed tomography did not show any acute intracranial findings. Magnetic resonance imaging of the brain showed an interval increase in the size of the right centrum semi-ovale and a hyperintense demyelinating lesion without enhancement which ruled out an acute demyelinating process (Figure 2).
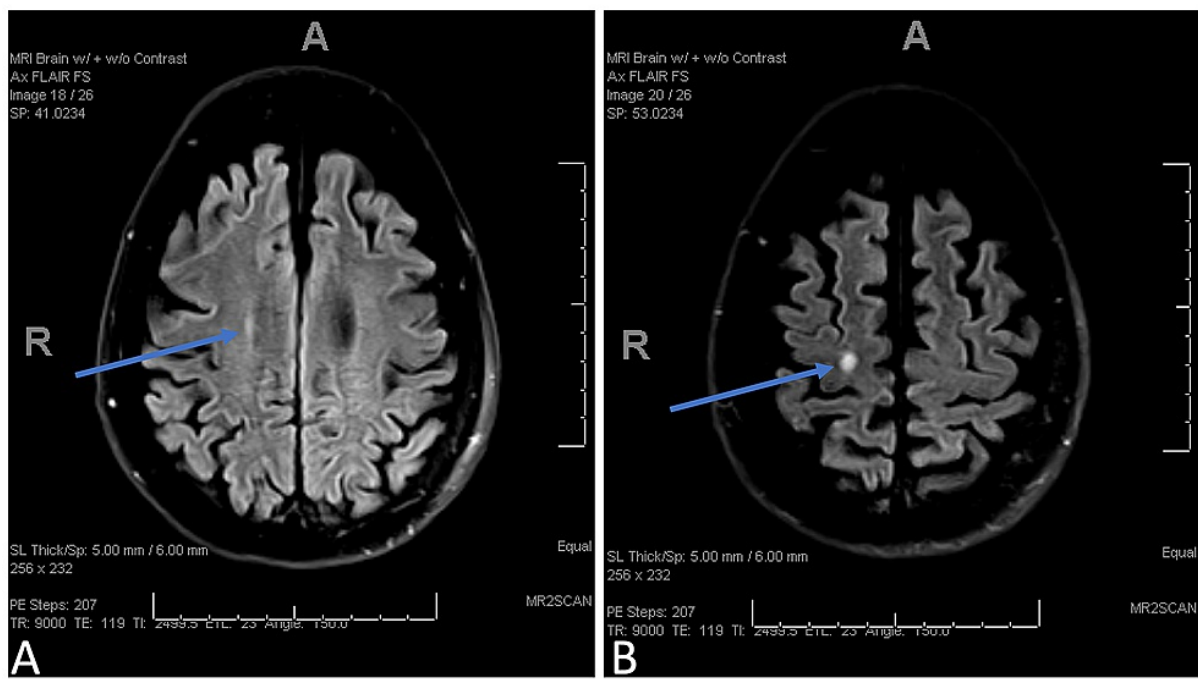

FIGURE 2: Axial FLAIR MRI. (A, B) Right centrum semi-ovale with hyperintense demyelinating lesion without enhancement (blue arrows).

FLAIR, fluid-attenuated inversion recovery; MRI, magnetic resonance imaging 


\section{Cureus}

She continued to become more lethargic over the next few hours and developed jerking of the left upper extremity and face. Medical management to treat the seizures was partially successful. Serum cefepime levels were measured and found to be elevated at $295 \mathrm{mg} / \mathrm{L}$. After three hours of hemodialysis, cefepime levels improved from $295 \mathrm{mg} / \mathrm{L}$ to $70 \mathrm{mg} / \mathrm{L}$. She was then started on continuous renal replacement therapy (CRRT) for ongoing clearance of cefepime. Serum cefepime level the next day was $17 \mathrm{mg} / \mathrm{L}$. Her mental status continued to improve and CRRT was stopped 48 hours after initiation. Figure 3 shows the trend of the patient's renal function during her first admission.

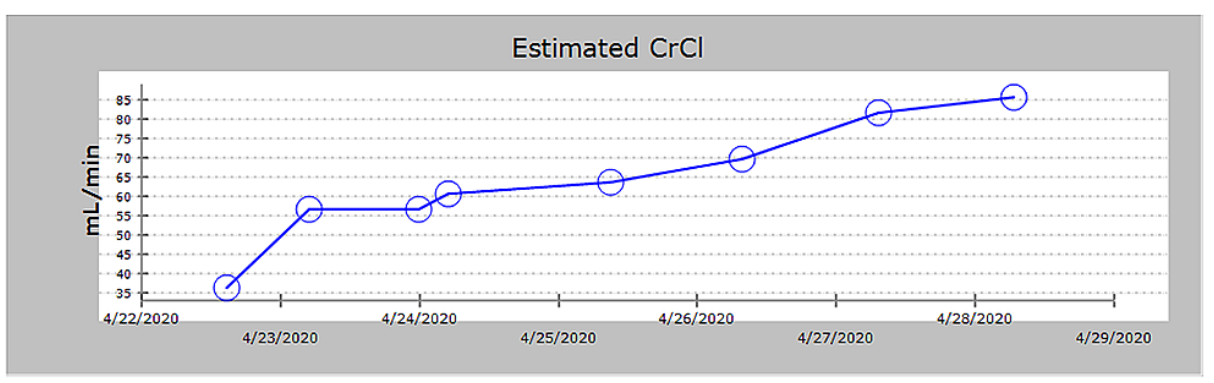

FIGURE 3: Trend of $\mathrm{CrCl}(\mathrm{mL} / \mathrm{min})$ during first admission.

$\mathrm{CrCl}$, creatinine clearance

Figure 4 shows the timeline of renal function in relation to her current admission. She did not require additional dialysis while hospitalized.

$$
\begin{aligned}
& \mathrm{Cr} 0.9, \text { eGFR }>60 \\
& \mathrm{CrCl}-56.5
\end{aligned}
$$

$1^{\text {st }}$ dose of Cefepime
Day -10
$4 / 23 / 20$

Last dose of Cefepime
Day - 1
$5 / 2 / 20$

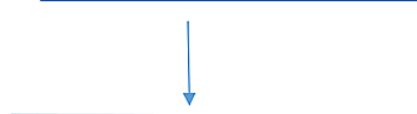

\begin{tabular}{|l|}
\hline \multicolumn{1}{|c|}{$2^{\text {nd }}$ Admission, Day 0} \\
$5 / 3 / 20$ \\
\hline $\mathrm{Cr} 2.19$, eGFR 23 \\
$\mathrm{CrCl}-$ not calculated \\
\hline
\end{tabular}

FIGURE 4: Timeline of creatinine and $\mathrm{CrCl}$ in relation to admission.

$\mathrm{CrCl}$, creatinine clearance (mL/min); Cr, Creatinine (mg/dL); eGFR, estimated glomerular filtration rate $\left(\mathrm{mL} / \mathrm{min} / 1.73 \mathrm{~m}^{2}\right)$

\section{Discussion}

Elevated serum cefepime level is an essential predictor of neurotoxicity, with a trough $>20 \mathrm{mg} / \mathrm{L}$ associated with a five-fold higher risk for neurologic events [3]. For this reason, a trough $>20 \mathrm{mg} / \mathrm{L}$ should generally be avoided. Predisposing factors of cefepime-induced neurotoxicity include excessive dosing, reduced renal function, increased cerebral penetration, and increased serum antibiotic levels [4].

While it is stated that less than $1 \%$ of patients experience neurotoxic side effects [5], recent literature has noted up to a 10-fold increase in the incidence of cefepime-induced neurotoxicity [6]. The increased incidence is likely due to increased antibiotic use, increased drug resistance [7], and increased symptom recognition [6]. A systematic review published in 2017 showed that up to $15 \%$ of all intensive care unit 
patients treated with cefepime experienced neurotoxic effects including encephalopathy, seizures, and coma, as well as subtler side effects such as depressed consciousness [1]. The study also showed that $26 \%$ of the patients experienced neurotoxic symptoms despite proper dosing and $20 \%$ of patients did not frequently experience renal dysfunction prior to the neurotoxic episode [1].

This also highlights the importance of antibiotic stewardship and careful monitoring of signs of cefepimeinduced neurotoxicity while following renal function. It involves dosing antibiotics carefully based on estimated $\mathrm{CrCl}$. This is especially valuable in hospitalized patients with AKI where eGFR might not be a reliable indicator of true eGFR, as eGFR equations are applicable for steady state. Kinetic eGFR was not calculated in this case. Her underlying chronic kidney disease, as revealed by the biopsy, likely played a significant role in predisposing to frequent AKIs (current and previous admission) and reduced renal clearance of the drug, and hence, precipitating acute cefepime neurotoxicity.

AKI is likely explained by acute tubular necrosis secondary to septic shock with need of vasopressors. Improvement in her neurological status after dialysis with reduction in serum cefepime level puts our diagnosis on a firm footing. Figure 5 shows the timeline of the events of dialysis in relation to cefepime levels.

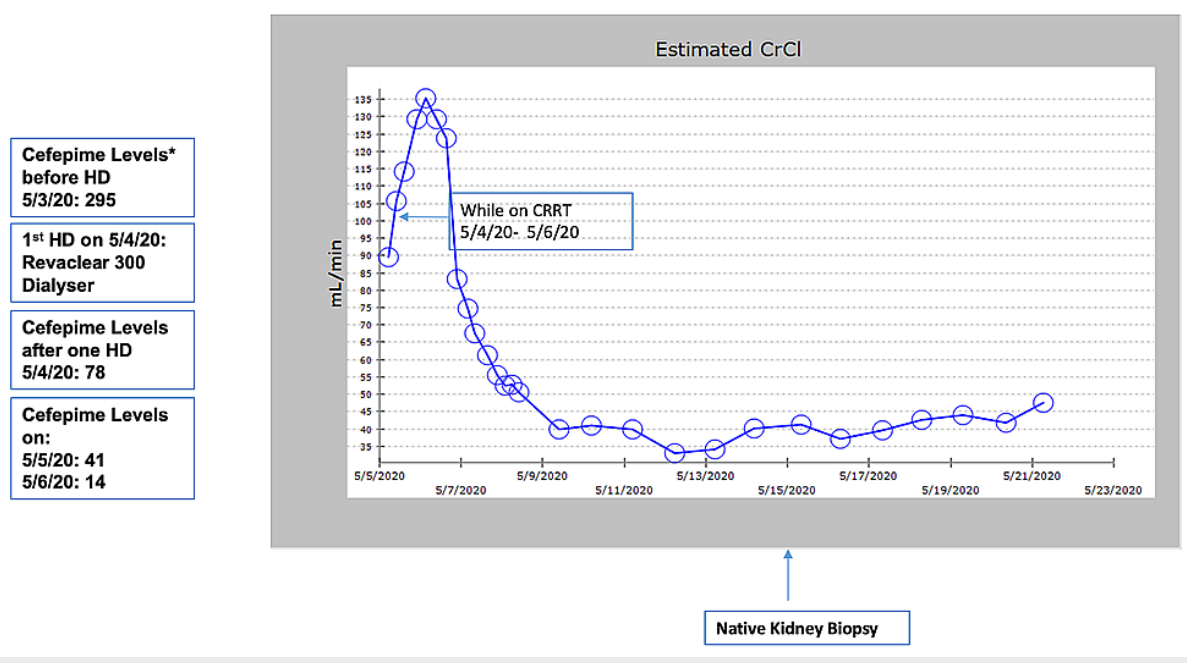

FIGURE 5: Trend of $\mathrm{CrCl}$ during current admission.

${ }^{*}$ All cefepime levels are in $\mathrm{mg} / \mathrm{L}$

$\mathrm{HD}$, hemodialysis; $\mathrm{CrCl}$, estimated creatinine clearance (mL/min); CRRT, continuous renal replacement therapy

\section{Conclusions}

To decrease the incidence of cefepime-induced neurotoxicity, treating patients with antibiotics such as ceftriaxone or cefotaxime can be considered due to their hepatic excretion. If cefepime must be administered, electroencephalogram (EEG) should strongly be considered in any patient with an acute change in mental status. This highlights the need to be vigilant in recognizing clinical signs and symptoms early. By monitoring the patient's mental status, utilizing EEG, measuring cefepime levels, and tracking renal function, adverse consequences can be recognized quickly and managed appropriately. Renal function should be monitored closely with monitoring of urine output, $\mathrm{CrCl}$, and kinetic eGFR. In patients with severe neurotoxic symptoms, hemodialysis may be employed to rapidly reduce antibiotic levels in the serum and antiepileptic drugs should be administered. Dosing of antiepileptic drugs should be adjusted in relation to the timing of dialysis to maintain therapeutic levels.

\section{Additional Information \\ Disclosures}

Human subjects: Consent was obtained or waived by all participants in this study. Conflicts of interest: In compliance with the ICMJE uniform disclosure form, all authors declare the following: Payment/services info: All authors have declared that no financial support was received from any organization for the submitted work. Financial relationships: All authors have declared that they have no financial relationships at present or within the previous three years with any organizations that might have an interest in the submitted work. Other relationships: All authors have declared that there are no other relationships or activities that could appear to have influenced the submitted work. 


\section{Cureus}

\section{References}

1. Payne LE, Gagnon DJ, Riker RR, Seder DB, Glisic EK, Morris JG, Fraser GL: Cefepime-induced neurotoxicity: a systematic review. Crit Care . 2017, 21:276. 10.1186/s13054-017-1856-1

2. Sugimoto M, Uchida I, Mashimo T, et al.: Evidence for the involvement of GABA(A) receptor blockade in convulsions induced by cephalosporins. Neuropharmacology. 2003, 45:304-314. 10.1016/s00283908(03)00188-6

3. Huwyler T, Lenggenhager L, Abbas M, et al.: Cefepime plasma concentrations and clinical toxicity: a retrospective cohort study. Clin Microbiol Infect. 2017, 23:454-459. 10.1016/j.cmi.2017.01.005

4. Lam S, Gomolin IH: Cefepime neurotoxicity: case report, pharmacokinetic considerations, and literature review. Pharmacotherapy. 2006, 26:1169-1174. 10.1592/phco.26.8.1169

5. Cefepime: drug information. (2020). Accessed: October 13, 2020: https://www-uptodatecom.medjournal.hmc.psu.edu:2200/contents/cefepime-drug-information? search=cefepime \&source=panel_....

6. Lamoth F, Buclin T, Pascual A, et al.: High cefepime plasma concentrations and neurological toxicity in febrile neutropenic patients with mild impairment of renal function. Antimicrob Agents Chemother. 2010, 54:4360-4367. 10.1128/AAC.01595-08

7. Chow KM, Szeto CC, Hui AC, et al.: Retrospective review of neurotoxicity induced by cefepime and ceftazidime. Pharmacotherapy. 2003, 23:369-373. 10.1592/phco.23.3.369.32100 